\section{Commentary: Pediatric myocardial recovery with a ventricular assist device: "Chance favors the prepared mind"}

\author{
Fumiya Yoneyama, MD, Susan Denfield, MD, and \\ Iki Adachi, MD
}

Despite extensive investigations, ${ }^{1}$ cardiac recovery with ventricular assist device (VAD) support remains a mystery. In this issue of the Journal, Philip and colleagues ${ }^{2}$ describe their experience of 2 infants with pulsatile biVAD support that resulted in sustained cardiac recovery. As the authors emphasize, myocardial recovery in children is rare. Interestingly, the recovery rate reported in the PediMACS registry (age $<19$ years) is even lower than that reported in the INTERMACS registry (age $\geq 19$ years), which completely contradicts the anecdotal wisdom established in the adult population of "the younger the patient, the greater the chance of recovery." ${ }^{, 3}$ Does the potential for cardiac recovery have a curious unimodal distribution with a peak at age 19? Alternatively, are pediatric clinicians not seeing as much recovery as they should, due to the mindset of just getting to transplantation while on VAD support, turning a blind eye to the possibility of recovery? This report could elicit fierce debate on this controversial matter.

The critical contribution of this report is to emphasize the importance of an active surveillance protocol to avoid overlooking an "unexpected" recovery. If the singular focus of VAD support is just rushing to heart transplantation, which

From Congenital Heart Surgery, Michael E. DeBakey Department of Surgery, Texas Children's Hospital, Baylor College of Medicine, Houston, Tex.

Disclosures: Dr Adachi serves as a consultant/proctor for Berlin Heart, Medtronic, Jarvik, BiVACOR, and Sony-Olympus Medical Solutions. All other authors reported no conflicts of interest.

The Journal policy requires editors and reviewers to disclose conflicts of interest and to decline handling or reviewing manuscripts for which they may have a conflict of interest. The editors and reviewers of this article have no conflicts of interest.

Received for publication April 27, 2020; revisions received April 27, 2020; accepted for publication April 30, 2020; available ahead of print May 11, 2020.

Address for reprints: Iki Adachi, MD, Congenital Heart Surgery, Texas Children's Hospital, Michael E. DeBakey Department of Surgery, Baylor College of Medicine, 6651 Main St, Houston, TX 77030 (E-mail: iadachi@bcm.edu).

JTCVS Techniques 2021;5:93-4

2666-2507

Copyright (C) 2020 The Authors. Published by Elsevier Inc. on behalf of The American Association for Thoracic Surgery. This is an open access article under the CC BY-NCND license (http://creativecommons.org/licenses/by-nc-nd/4.0/).

https://doi.org/10.1016/j.xjtc.2020.04.024

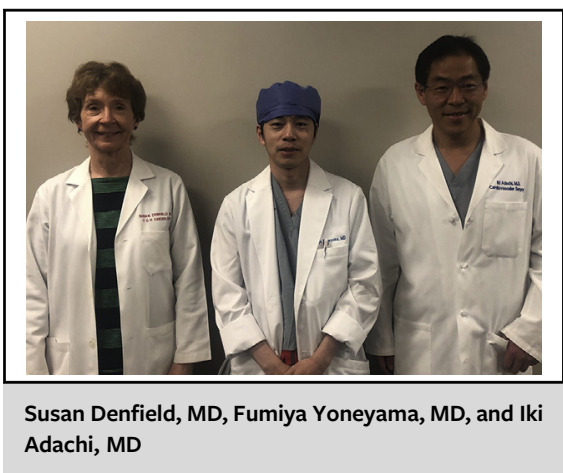

CENTRAL MESSAGE

Myocardial recovery with ventricular assist device support is the silver lining in the cloud of heart failure support in children. An active surveillance of cardiac function may allow greater recognition of recovery.

is the current clinical reality in the pediatric field, ${ }^{5}$ early (and subtle) signs of rare events (ie, recovery) may be missed. Active routine surveillance would keep the clinician's mind open to these rare events. Given the substantially shorter waitlist time in children with VAD support compared with their adult counterparts, the risk of missing opportunities is significantly greater in the pediatric population. ${ }^{6}$ To paraphrase D.H. Lawrence, the eyes don't see what the mind doesn't seek. ${ }^{7}$ The patients in the present cohort were lucky enough not to have received an organ offer before being inactivated on the transplantation wait-list due to the recognition of cardiac recovery.

To induce cardiac recovery, not as luck but as an intended therapeutic target, implementation of an observation period (with waitlist inactivation and active surveillance) following VAD implantation is essential. The current lack of an observation period could explain the counterintuitively low incidence of cardiac recovery seen in children. ${ }^{6}$ The negative impact of the standard "rushing to transplantation" approach on posttransplantation outcomes is becoming increasingly evident. ${ }^{8}$ While acknowledging the risk of VAD complications, a "watchful waiting" strategy may lead to a higher incidence of recovery by avoiding premature transplantation resulting from the failed realization of recovery potential, while acknowledging the risk of VAD complications. With the ongoing reduction of VAD-related risks, waiting, as 
the preferred approach, may be more attractive to pediatric centers that currently "rush to transplant."

"Chance favors the prepared mind" (Louis Pasteur, 1854). Keeping an open mind may be the key to achieving increased pediatric myocardial recovery, from an unexpected surprise that is rarely recognized to an intended goal to attain. Philip and colleagues should be congratulated for their effort to shed light on this important topic.

\section{References}

1. Drakos SG, Mehra MR. Clinical myocardial recovery during long-term mechanical support in advanced heart failure: insights into moving the field forward. $J$ Heart Lung Transplant. 2016;35:413-20.

2. Philip J, Machado D, Shihm R, Shenoy A, Ebraheem M, Sullivan K, et al. Myocardial recovery following pulsatile biventricular assist device support in infants: report of 2 cases. J Thorac Cardiovasc Surg Tech. 2021;5:89-92.
3. Topkara VK, Garan AR, Fine B, Godier-Furnémont AF, Breskin A, Cagliostro B, et al. Myocardial recovery in patients receiving contemporary left ventricular assist devices: results from the interagency registry for mechanically assisted circulatory support (INTERMACS). Circ Heart Fail. 2016;9:e003157.

4. Goldstein DJ, Maybaum S, MacGillivray TE, Moore SA, Bogaev R, Farrar DJ, et al. Young patients with nonischemic cardiomyopathy have higher likelihood of left ventricular recovery during left ventricular assist device support. $J$ Card Fail. 2012;18:392-5.

5. Adachi I. Pediatric ventricular assist device support as a permanent therapy: clinical reality. J Thorac Cardiovasc Surg. 2019;158:1438-41.

6. Adachi I, Zea-Vera R, Tunuguntla H, Denfield SW, Elias B, John R, et al. Centrifugal-flow ventricular assist device support in children: a single-center experience. J Thorac Cardiovasc Surg. 2019;157:1609-17.e2.

7. Lawrence DH. Lady Chatterley's Lover. 1st ed. Italy: Tipografia Giuntina; 1928.

8. Riggs KW, Zafar F, Lorts A, Villa CR, Bryant R III, Morales DLS. Optimizing postcardiac transplantation outcomes in children with ventricular assist devices: how long should the bridge be? ASAIO J. September 39, 2019 [Epub ahead of print]. 